\title{
Event reconstruction of free-streaming data for the RICH detector in the CBM experiment
}

J. Adamczewski-Musch ${ }^{1}$, P. Akishin ${ }^{7}$, K.-H. Becker ${ }^{2}$, J. Bendarouach ${ }^{3}$, C. Deveaux ${ }^{3}$, M. Dürr ${ }^{3}$, J. Eschke ${ }^{1}$, J. Förtsch ${ }^{2}$, J. Heep ${ }^{3}$, C. Höhne ${ }^{3,1}$, D. Ivanishchev ${ }^{4}$, K.H. Kampert ${ }^{2}$, L. Kochenda ${ }^{4,5}$, P. Kravtsov ${ }^{4,5}$, I. Kres ${ }^{2}$, S. Lebedev ${ }^{3,7, *}$, E. Lebedeva ${ }^{3}$,

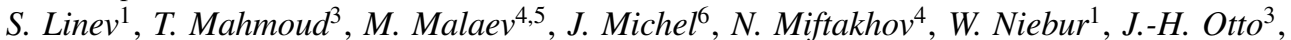
E. Ovcharenko ${ }^{3,7}$, V. Patel ${ }^{2}$, C. Pauly ${ }^{2}$, D. Pfeifer ${ }^{2}$, Y. Riabov $^{4}$, E. Roshchin $^{4}$, V. Samsonov ${ }^{4,5,8}$, O. Tarasenkova ${ }^{4}$, M. Traxler ${ }^{1}$, D. Tyts ${ }^{4}$, M. Vznuzdaev ${ }^{4}$, A.A. Weber ${ }^{3}$, and P. Zumbruch ${ }^{1}$

${ }^{1}$ GSI Helmholtzzentrum für Schwerionenforschung GmbH, D-64291 Darmstadt, Germany

${ }^{2}$ Department of Physics, University of Wuppertal, D-42097 Wuppertal, Germany

${ }^{3}$ Institute of Physics II and Institute of Applied Physics, Justus Liebig University Giessen, D-35392 Giessen, Germany

${ }^{4}$ National Research Centre "Kurchatov Institute" B.P.Konstantinov Petersburg Nuclear Physics Institute, 188300 Gatchina, Russia

${ }^{5}$ National Research Nuclear University MEPhI (Moscow Engineering Physics Institute), 115409, Moscow, Russia

${ }^{6}$ Institut für Kernphysik, Göthe University Frankfurt, D-60438 Frankfurt am Main, Germany

${ }^{7}$ Laboratory of Information Technologies, Joint Institute for Nuclear research (JINR-LIT), Dubna, Russia

${ }^{8}$ St. Petersburg Polytechnic University (SPbPU), St. Petersburg, Russia

\begin{abstract}
The Compressed Baryonic Matter (CBM) experiment is a dedicated heavy ion collision experiment at the FAIR facility. It will be one of the first HEP experiments which works in a triggerless mode: data received in the DAQ from the detectors will not be associated with events by a hardware trigger anymore. All raw data within a given time period will be collected continuously in containers, so-called time-slices. The task of the reconstruction algorithms is to create events out of this raw data stream. In this contribution, the optimization of the reconstruction software in the RICH detector to the free-streaming data flow is presented. The implementation of ring reconstruction algorithms which use time measurements of the hits as an additional parameter is discussed.
\end{abstract}

\section{The Compressed Baryonic Matter (CBM) experiment}

The Compressed Baryonic Matter (CBM) experiment at the future FAIR facility will investigate the phase diagram of strongly interacting matter at high net-baryon density and moderate temperature in proton-proton and nucleus-nucleus collisions. The SIS100 synchrotron will provide proton beam with energies up to $29 \mathrm{GeV}$ and up to $11 \mathrm{AGeV}$ for the heaviest nuclei. The beam extracted to the CBM cave reaches intensities up to $10^{9} \mathrm{Au}$ ions per second.

One key item of the CBM physics program is the precise measurement of electromagnetic radiation by reconstructing low-mass vector mesons and $J / \psi$ via the di-electron decay

\footnotetext{
${ }^{*}$ Corresponding author, e-mail: s.lebedev@gsi.de
} 
channel. This measurement requires efficient and clean electron identification, and in particular $e / \pi$ separation, in wide acceptance. Electron identification and suppression of pions will be performed by a Ring Imaging Cherenkov detector (RICH) in combination with several Transition Radiation detectors (TRD) which are both positioned behind the Silicon Tracking System (STS). Figure 1 shows a sketch of the CBM detector setup. A detailed overview of the CBM physics program is given in [1].

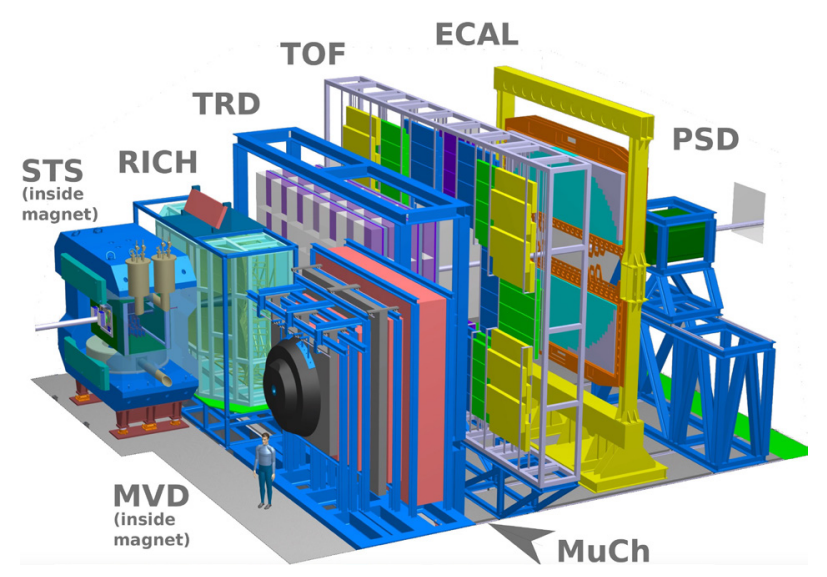

Figure 1. Sketch of the CBM experimental setup. From left to right: Micro-vertex Detector (MVD) and Silicon Tracking System (STS) inside a dipole magnet, Ring Imaging Cherenkov (RICH) detector, Transition Radiation Detectors (TRD), Time-of-flight wall (TOF), Electromagnetic CALorimeter (ECAL) and Projectile Spectator Detector (PSD). The muon detector (MUCH) is shown in its parking position; it can be interchanged with the RICH detector.

\section{Ring Imaging CHerenkov ( $\mathrm{RICH}$ ) detector}

The RICH detector (see Figure 2) is designed to provide electron identification covering the momentum range up to $8 \mathrm{GeV} / \mathrm{c}$. It is split into two identical symmetrical parts below and above the beam pipe with two spherical mirror planes and two photon detector planes. $\mathrm{CO}_{2}$ gas at 2 mbar overpressure $\left(\gamma_{t h r}=33, p_{t h r}=4.65 \mathrm{GeV} / \mathrm{c}\right)$ is used as radiator. Each of the two mirror planes consists of 40 spherical mirror tiles with 3 m radius of curvature and spectral reflectivity down to the $\mathrm{UV}$ range $(\mathrm{Al}+\mathrm{MgF} 2$ reflective coating). The Cherenkov photons are detected using two photon detector planes $\approx 2 \times 0.6 \mathrm{~m}^{2}$ each, together equipped with about 1000 Hamamatsu H12700 Multianode Photomultiplier tubes. They are placed inside iron shielding boxes in order to reduce the magnetic stray field (aim at $<1 \mathrm{mT}$ at the photocathode). Beam test results with a real-size length RICH prototype showed that around 25 photons are detected per electron ring. A detailed technical description of the CBM RICH can be found in the Technical Design Report [2], and in [3-7].

Figure 3 shows an example of a typical response of the RICH detector to a central $\mathrm{Au}$ $A u$ collision at $8 \mathrm{AGeV}$ beam energy. Around 25 rings per event are registered which gives about 630 hits per event on average. The most significant fraction of recorded photons in the RICH stems from $e^{ \pm}$from $\gamma$-conversions in the STS detector or the magnet yoke (17 rings per event). 

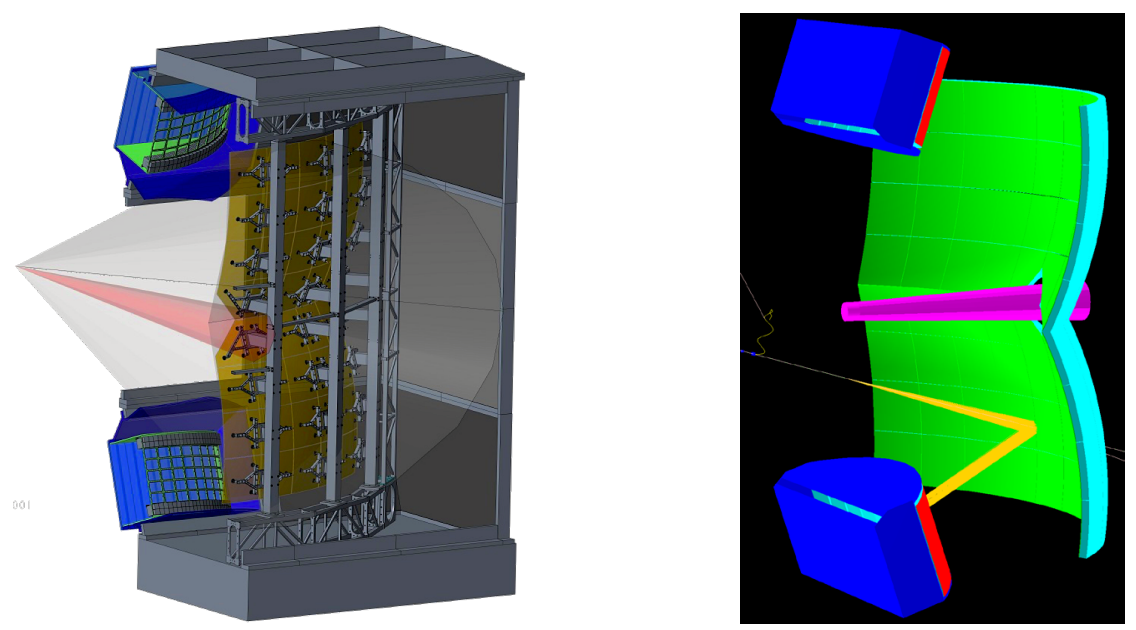

Figure 2. Left: The mechanical design of the RICH detector. Right: The RICH detector in the $C B M$ ROOT framework simulation (support structure is not shown). The Cherenkov photons emitted by an electron are shown in orange color. They are reflected by the mirror (green) onto the photon detector plane (red).
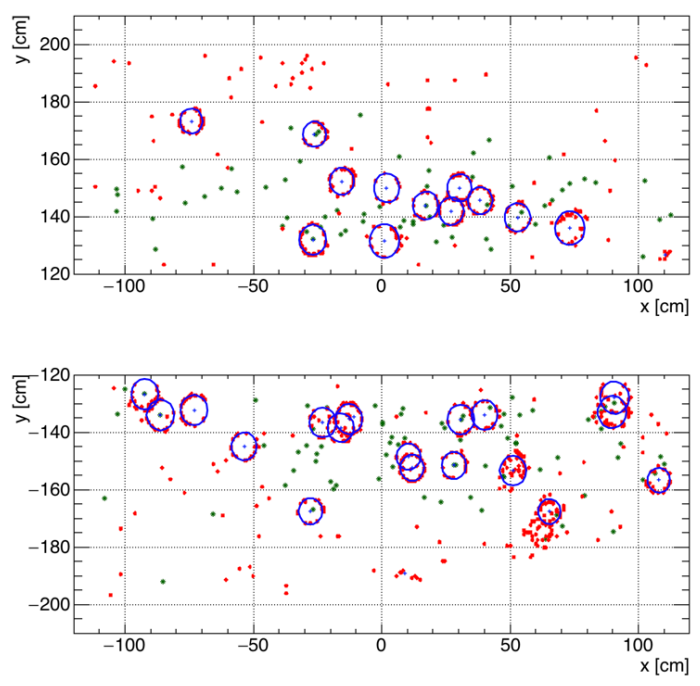

Figure 3. Simulation of a typical response of the RICH detector to a central $A u-A u$ collision at $8 \mathrm{AGeV}$ beam energy. Blue circles: reconstructed rings; red points: RICH hits including noisy channels; green markers: reconstructed track projections onto the photon detector plane.

\section{Free streaming data}

The CBM experiment will be one of the first HEP experiments which works in a triggerless mode: data received from the detectors are not associated with events by a hardware trigger anymore. For the highest interaction rate $(10 \mathrm{MHz})$ this will result in the fact that data from 
different collisions may overlap in time. All raw data within a given time period are collected continuously in containers, so-called time-slices. The reconstruction algorithms take timeslices as input data.
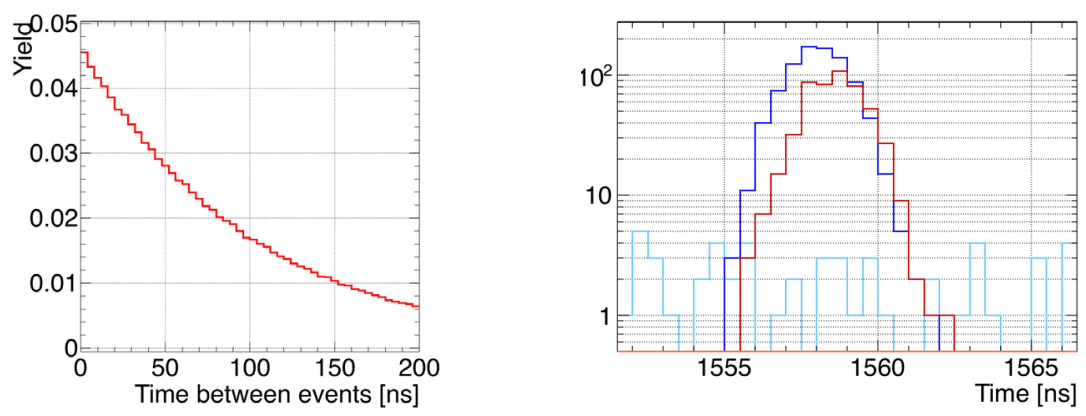

Figure 4. Left: distribution of times between two neighboring events. Right: distribution of the number of RICH hits in time for two overlapping events. Red and blue colors correspond to two different events; light blue color corresponds to noise hits.

In the simulation the start time of an individual event is generated using the Poisson process. The left plot of Figure 4 shows the distribution of the times between two neighboring events for $10 \mathrm{MHz}$ interaction rate. About $10 \%$ of collisions happen within a $10 \mathrm{~ns}$ interval which results in overlapping events in time. The right plot of Figure 4 shows the distribution of hit times for two overlapping events in the RICH detector.

A time stamp of a RICH hit is the sum of the event time, the time of flight from the collision point to the detector, and a measurement error of the detector. For the Cherenkov photons originating from signal $e^{ \pm}$the time of flight until the photon detector plane equals to around $19 \mathrm{~ns}$ and is almost the same for all photons. The time resolution of the photon detector was obtained after several lab and in beam tests and is expected to be less then $1 \mathrm{~ns}$. In the simulation each RICH hit time is smeared according to a Gaussian distribution with $\sigma=1 \mathrm{~ns}$.

The dark rate is one of the sources of noise hits in the RICH detector. In the simulation the dark noise of individual pixels is set to $1 \mathrm{kHz}$ which is rather on the large side as seen from the measurements [8]. One can see these noise hits (light blue color) in the right plot of Figure 4. The event correlated noise depends on the event multiplicity. The pixel dead time is set to $50 \mathrm{~ns}$.

Figure 5 shows the initial distribution of RICH hits in time for $A u$ - $A u$ minimum bias collisions at $8 \mathrm{AGeV}$ beam energy assuming $10 \mathrm{MHz}$ interaction rate. This is only a part of a time-slice with about 100 events.

\section{Ring reconstruction algorithm}

The developed ring recognition algorithm is an updated version of the algorithm which works in the event-by-event mode without the usage of time information [9-14]. The updated algorithm is also standalone and uses only RICH hit information ( $X, Y$ and time measurements) as input. No track information is used.

The ring reconstruction begins with the ring-candidate search which is based on the Hough Transform where hit triplets are combined, and the corresponding ring parameters 

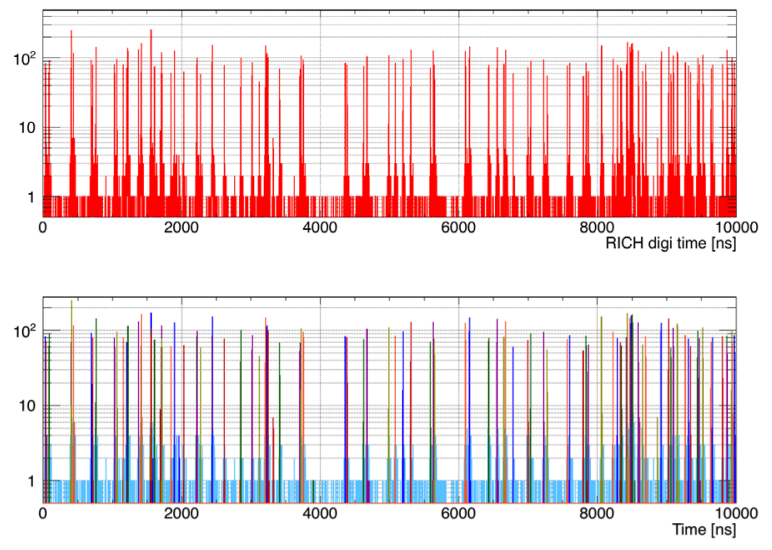

Figure 5. Distribution of the number of RICH hits in time for a part of a time-slice with about 100 $A u$ - $A u$ minimum bias collisions at $8 \mathrm{AGeV}$ beam energy assuming $10 \mathrm{MHz}$ interaction rate. Top: All events are shown in red color. Bottom: Each color corresponds to an individual event. Light blue color corresponds to the noise hits.

are calculated. Instead of combining all possible hit triplets in the whole photon detector plane, only hits within a predefined local area around the initial hit are selected and additionally only hits within $3 \sigma$ (detector time resolution: $1 \mathrm{~ns}$ ) time window are selected. The time window value is a reasoned assumption because the difference in time of flight of photons from one ring is negligible in comparison to the detector time resolution. Thus the triplets are built only for a small fraction of selected hits which makes the whole procedure very fast. For each triplet the corresponding ring parameters $(X, Y, R)$ are calculated and Hough histograms are filled. Analyzing the peaks in histograms one either accepts ring-candidate or rejects them.

The next step is to filter the ring-candidate array and reject all fake ring-candidates which are formed by random combinations of hits. For each ring a quality value is derived by an artificial neural network from 6 input parameters: number of hits in the ring, ring radius, $\chi^{2}$ of the circle fit, ring position on the photon detector plane, uniformity of the hit distribution around the ring, and the number of hits in a small corridor around the ring fit. Then the following rule is applied: the ring-candidate is rejected if it shares more than $25 \%$ of its hits with a better quality ring.

For all found rings the time measurement is calculated as the average time of all hits belonging to the ring.

\section{Results}

All results presented in this section are based on simulation studies performed for $A u-A u$ minimum bias collisions from the Ultrarelativistic Quantum Molecular Dynamics model (UrQMD) [15] at $8 \mathrm{AGeV}$ beam energy assuming $10 \mathrm{MHz}$ interaction rate. In order to enhance statistics and investigate the RICH response to primary electrons, $5 e^{+}$and $5 e^{-}$were embedded into each UrQMD event at the primary vertex.

The initial distribution of RICH hits and reconstructed rings in time is shown in Figure 6. The reconstructed rings clearly represent groups, corresponding to events. 


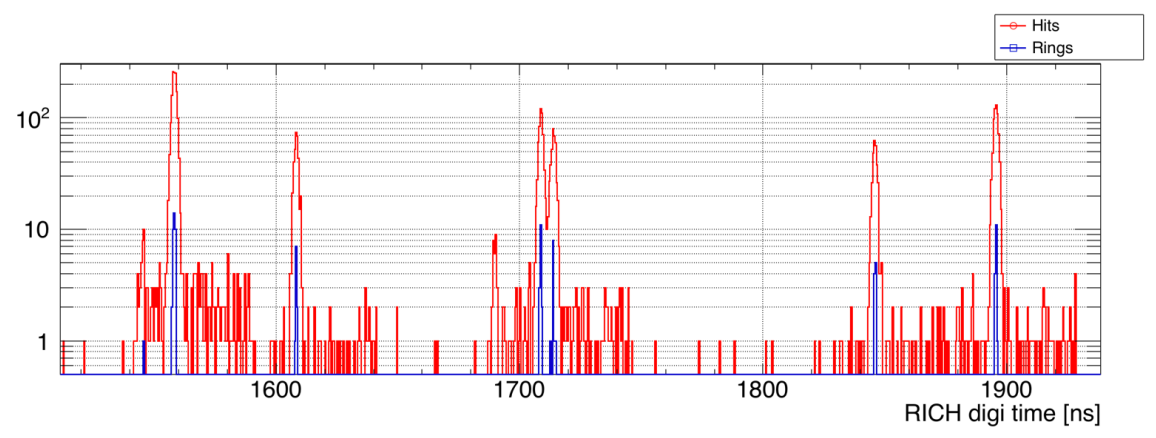

Figure 6. Distribution of the number of RICH hits (red) in time and the number of reconstructed rings (blue) in time. Only a part of a time-slice is shown. Simulation: $A u-A u$ minimum bias collisions at $8 \mathrm{AGeV}$ beam energy, $10 \mathrm{MHz}$ interaction rate.

Figure 7 shows the ring reconstruction efficiency for primary $e^{ \pm}$in dependence on momentum (left plot) and in dependence on track multiplicity in an event (right plot). Results are presented for rings from primary $e^{ \pm}$with at least 7 detected photons and rings from for socalled primary reference $e^{ \pm}$which have more than 15 detected photons in the ring. The ring reconstruction efficiency integrated over the momentum range from $0-12 \mathrm{GeV} / \mathrm{c}$ is $96.5 \%$ for primary $e^{ \pm}$and $97.7 \%$ for the reference set. The corresponding number of fake rings is around $1 \%$.
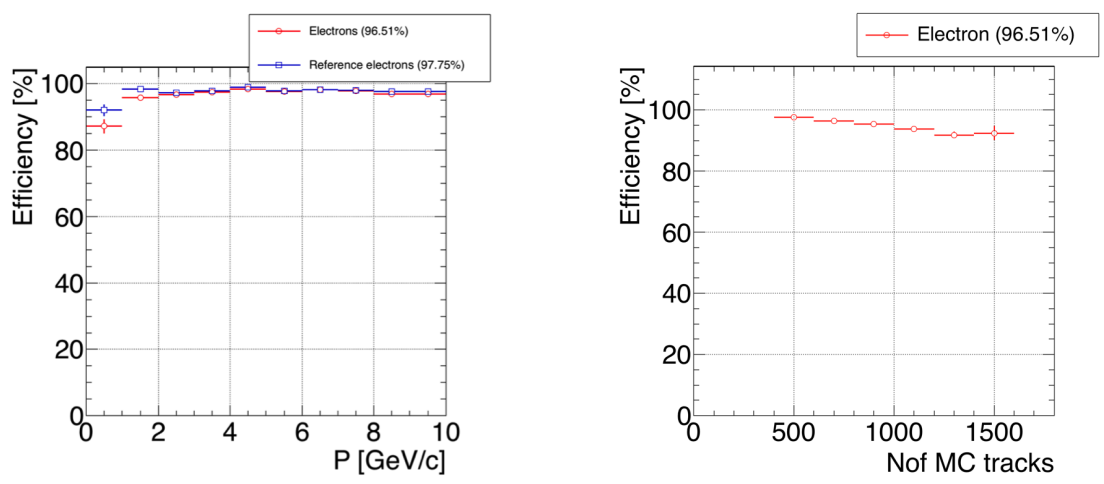

Figure 7. Left: Ring reconstruction efficiency for primary $e^{ \pm}$(red circle) and primary reference $e^{ \pm}$(blue square) in dependence on momentum. Right: Ring reconstruction efficiency for primary $e^{ \pm}$in dependence on track multiplicity (number of Monte-Carlo tracks in event). Simulation: $A u-A u$ minimum bias collisions at $8 \mathrm{AGeV}$ beam energy, $10 \mathrm{MHz}$ interaction rate.

Figure 8 shows the ring reconstruction efficiency for primary $e^{ \pm}$in dependence on the interaction rate. The performance of the ring reconstruction algorithm does not depend on the interaction rate and is the same as the event-by-event reconstruction performance.

To summarize, the time-based reconstruction for the RICH detector were developed and showed good performance in the simulation studies. The first test on a real data is expected for the mCBM experiment setup in 2019. 


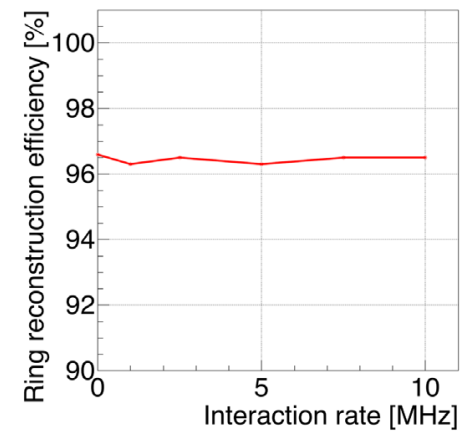

Figure 8. Ring reconstruction efficiency for primary $e^{ \pm}$in dependence on interaction rate. "Zero" interaction rate corresponds to the event-by-event mode. Simulation: $A u-A u$ minimum bias collisions at $8 \mathrm{AGeV}$ beam energy.

\section{Acknowledgments}

This work was supported by the Hessian LOEWE initiative through the Helmholtz International Center for FAIR (HIC for FAIR), by the Helmholtz Graduate School for Hadron and Ion Research, by the GSI F\&E-Cooperations with Giessen and Wuppertal (WKAMPE1012), by BMBF Grants 05P12RGFCG, 05P12PXFCE, 05P09PXFC5, 05P15PXFCA, and 05P15RGFCA, by Helmholtz Grant IK-RU-002, by SCROSATOM through FRRC, and by the Ministry of Education and Science of the Russian Federation (grant no. 14.A12.31.0002) in accordance with the Russian Federation Government Regulation no. 220.

\section{References}

[1] B. Friman, et al., (Eds.), The CBM Physics Book: Compressed Baryonic Matter in Laboratory Experiments (Springer Series: Lecture Notes in Physics, vol. 814, 2011)

[2] C. Höhne (Ed.) et al., CBM-RICH Technical Design Report, GSI-2014-00528, http://repository.gsi.de/record/65526.

[3] C. Hoehne et al. NIM A 595187 (2008)

[4] C. Hoehne et al. NIM A 639294 (2011)

[5] J. Adamczewski-Musch et al. NIM A 766101 (2014)

[6] J. Adamczewski-Musch et al. NIM A 876 65-68 (2017)

[7] J. Adamczewski-Musch et al. NIM A 845 434-438 (2017)

[8] J. Adamczewski-Musch et al. NIM A 876 123-125 (2017)

[9] S. Lebedev et al, J. Phys.: Conf. Series 219032015 (2010)

[10] S. Lebedev et al, J. Phys.: Conf. Series 396022029 (2012)

[11] S. Lebedev et al, PoS ACAT2010 060 (2010)

[12] J. Adamczewski-Musch et al. NIM A 766250 (2014)

[13] G. Ososkov and N. Chernov, Comp. Phys. Comm. 33 329-333 (1984)

[14] N. Chernov, J. Math. Im. Vi. 27 231-239 (2007)

[15] S. Bass et al, Prog. Part. Nucl. Phys. 41 255-370 (1998) 